\title{
ASYMPTOTIC BEHAVIOR OF SOLUTIONS TO THE FULL COMPRESSIBLE NAVIER-STOKES EQUATIONS IN THE HALF SPACE*
}

\author{
FEIMIN HUANG ${ }^{\dagger}$, JING LI $^{\ddagger}$, AND XIAODING SHI ${ }^{\S}$
}

Abstract. The one-dimensional motion of compressible viscous and heat-conductive fluid is investigated in the half space. By examining the sign of fluid velocity prescribed on the boundary, initial boundary value problems with Dirichlet type boundary conditions are classified into three cases: impermeable wall problem, inflow problem and outflow problem. In this paper, the asymptotic stability of the rarefaction wave, boundary layer solution, and their combination is established for both the impermeable wall problem and the inflow problem under some smallness conditions. The proof is given by an elementary energy method.

Key words. Asymptotic behavior of solutions, Navier-Stokes equations, boundary layer, rarefaction wave.

AMS subject classifications. 35L65.

\section{Introduction}

The one-dimensional full compressible Navier-Stokes equations in Eulerian coordinate is given by

$$
\left\{\begin{array}{l}
\tilde{\rho}_{t}+(\tilde{\rho} \tilde{u})_{\tilde{x}}=0 \\
(\tilde{\rho} \tilde{u})_{t}+\left(\tilde{\rho} \tilde{u}^{2}+\tilde{p}\right)_{\tilde{x}}=\mu \tilde{u}_{\tilde{x} \tilde{x}} \\
\left(\tilde{\rho}\left(\tilde{e}+\frac{\tilde{u}^{2}}{2}\right)\right)_{t}+\left(\tilde{\rho} \tilde{u}\left(\tilde{e}+\frac{\tilde{u}^{2}}{2}\right)+\tilde{p} \tilde{u}\right)_{\tilde{x}}=\kappa \tilde{\theta} \tilde{x} \tilde{x}+\left(\mu \tilde{u} \tilde{u}_{\tilde{x}}\right)_{\tilde{x}}
\end{array}\right.
$$

where $\tilde{u}(\tilde{x}, t)$ denotes the velocity, $\tilde{\rho}(\tilde{x}, t)>0$ the density, $\tilde{\theta}(\tilde{x}, t)>0$ the absolute temperature, $\mu>0$ the viscosity constant, and $\kappa>0$ the coefficient of heat conduction. The pressure $\tilde{p}$, the internal energy $\tilde{e}$, and the entropy $\tilde{s}$ are functions of $\tilde{\rho}$ and $\tilde{\theta}$ or functions of $\tilde{v}$ and $\tilde{\theta}$, where $\tilde{v}=\frac{1}{\tilde{\rho}}$ is the specific volume. Since there are only two independent variables, $\tilde{p}$ and $\tilde{e}$ are also regarded as functions of $\tilde{v}$ and $\tilde{s}$. We denote

$$
\tilde{p}=\tilde{p}(\tilde{v}, \tilde{\theta})=\hat{p}(\tilde{v}, \tilde{s}), \tilde{e}=\tilde{e}(\tilde{v}, \tilde{\theta})=\hat{e}(\tilde{v}, \tilde{s}) .
$$

We assume that

$$
\begin{aligned}
& \tilde{p}_{\tilde{v}}<0, \tilde{e}_{\tilde{\theta}}>0, \hat{p}_{\tilde{v} \tilde{v}}>0, \\
& \hat{p}(\tilde{v}, \tilde{s}) \text { is a convex function of }(\tilde{v}, \tilde{s}),
\end{aligned}
$$

which together with the thermodynamic relation

$$
d \tilde{e}=\tilde{\theta} d \tilde{s}-\tilde{p} d \tilde{v}
$$

\footnotetext{
* Received: December 3 2007; accepted (in revised version): March 23, 2009.

$\dagger$ Institute of Applied Mathematics, AMSS, Academia Sinica, Beijing 100190, China (fhuang@ amt.ac.cn)

${ }^{\ddagger}$ Institute of Applied Mathematics, AMSS, Academia Sinica, Beijing 100190, China (ajingli@ gmail.com).

$\S$ Department of Mathematics, Graduate School of Science, Beijing University of Technology and Chemical, Beijing 100029, China (shixd@mail.buct.edu.cn).
} 
ensures the inviscid system of (1.1) is strictly hyperbolic and each characteristic field is either genuinely nonlinear or linearly degenerate. It is noted that the ideal polytropic gas satisfies this assumption (1.2).

We consider the motion of a gas in the half space $\tilde{x}>0$. Our initial data is

$$
(\tilde{\rho}, \tilde{u}, \tilde{\theta})(\tilde{x}, 0)=\left(\tilde{\rho}_{0}, \tilde{u}_{0}, \tilde{\theta}_{0}\right)(\tilde{x}) \rightarrow\left(\rho_{+}, u_{+}, \theta_{+}\right) \text {, as } \tilde{x} \rightarrow \infty .
$$

As pointed out by [8], boundary conditions of Dirichet type for (1.1) are classified into three cases:

Case 1. (zero velocity on the boundary):

$$
\tilde{u}(0, t)=u_{-}=0, \tilde{\theta}(0, t)=\theta_{-}, t>0,
$$

Case 2. (negative velocity on the boundary):

$$
\tilde{u}(0, t))=u_{-}<0, \tilde{\theta}(0, t)=\theta_{-}, t>0,
$$

Case 3. (positive velocity on the boundary):

$$
\tilde{u}(0, t)=u_{-}>0, \tilde{\rho}(0, t)=\rho_{-}, \tilde{\theta}(0, t)=\theta_{-}, t>0 .
$$

We assume that the initial data satisfies one of (1.5) as compatibility condition. It is noted that in cases 1 and 2, the density on the boundary can not be imposed, but in case 3 it has to be imposed so that the first equation of (1.1) is well posed. The initial boundary value problems $(1.1)-(1.5)_{1},(1.1)-(1.5)_{2}$, and $(1.1)-(1.5)_{3}$ are called the impermeable wall problem, outflow problem, and inflow problem respectively.

There have been many works on the asymptotic behavior of solutions to the Cauchy problem of the system (1.1). We refer to [3-4, 9-11] and the references therein. All these results showed that the large time behavior of the solutions are described by the corresponding Riemann solutions to the hyperbolic part of (1.1). Recently, the initial boundary value problem has attracted interest because it is more physical than the Cauchy problem. We refer to $[1-2,7-8,12-16]$. Most of these results are concerned with isentropic gas, where only the conservation of mass and momentum of (1.1) are considered. It was found that a new wave, boundary layer solution, appears in the solutions due to the boundary effect. To be more physical, we should consider the full compressible Navier-Stokes equation (1.1). Comparing with isentropic gas, the boundary conditions for the full compressible Navier-Stokes system become more complicated and analytically it is more difficult to control the terms coming from the boundary. More recently, Kawashima and Zhu [16] investigated the existence of the boundary layer solution and its asymptotic stability for the outflow problem of (1.1) in which the sign of the velocity $u_{-}$on the boundary is negative, which plays a key role in the a priori estimate. In this paper we focus our attention on the impermeable wall problem and inflow problem. We shall investigate the asymptotic stability of the rarefaction wave, the boundary layer solution and their combination. Unlike the outflow problem, the velocity $u_{-}$on the boundary for the inflow problem is positive which is not good for the analysis, we have to estimate more boundary terms than those of [16].

Let us turn to the original problem. To handle the system (1.1) more easily, we transform (1.1) into the Lagrangian coordinate $(x, t)$ for the impermeable wall 
problem and inflow problem, i.e.,

$$
\left\{\begin{array}{lr}
v_{t}-u_{x}=0, & x>k_{-} t, t>0 \\
u_{t}+p(v, \theta)_{x}=\mu\left(\frac{u_{x}}{v}\right)_{x} & x>k_{-} t, t>0 \\
\left(e(v, \theta)+\frac{u^{2}}{2}\right)_{t}+(p(v, \theta) u)_{x}=\left(\kappa \frac{\theta_{x}}{v}+\mu \frac{u u_{x}}{v}\right)_{x}, & x>k_{-} t, t>0
\end{array}\right.
$$

where $\rho(x, t)=\tilde{\rho}(\tilde{x}, t)$, etc., $\hat{p}=p(v, s)$, etc., $k_{-}=-\frac{u_{-}}{v_{-}}$, and the boundary condition $(1.5)_{1}$ and $(1.5)_{3}$ become, respectively:

Impermeable wall problem:

$$
u(0, t)=u_{-}=0, \theta(0, t)=\theta_{-}, t>0,
$$

Inflow problem:

$$
u\left(k_{-} t, t\right)=u_{-}>0, v\left(k_{-} t, t\right)=v_{-}, \theta\left(k_{-} t, t\right)=\theta_{-}, t>0,
$$

and the initial data is

$$
(v, u, \theta)(x, 0)=\left(v_{0}, u_{0}, \theta_{0}\right)(x) \rightarrow\left(v_{+}, u_{+}, \theta_{+}\right) \text {as } x \rightarrow \infty .
$$

Before stating our main results, we recall some properties of $p$, which is useful in the following. The second law of thermodynamics (1.3) yields if $p=p(v, \theta), e=e(v, \theta), s=$ $s(v, \theta)$,

$$
e_{v}=-\left(p-\theta p_{\theta}\right), \quad s_{v}=p_{\theta}, \quad s_{\theta}=\frac{e_{\theta}}{\theta}
$$

and if $p=\hat{p}(v, s), \theta=\hat{\theta}(v, s), e=\hat{e}(v, s)$,

$$
\hat{e}_{v}=-p, \hat{e}_{s}=\theta, \hat{p}_{v}=p_{v}-\frac{\theta p_{\theta}^{2}}{e_{\theta}}, \hat{p}_{s}=\frac{\theta p_{\theta}}{e_{\theta}}, \hat{\theta}_{v}=-\frac{\theta p_{\theta}}{e_{\theta}}, \quad \hat{\theta}_{s}=\frac{\theta}{e_{\theta}} .
$$

We now formulate our main results. It is known that the characteristic speeds of hyperbolic part of (1.6) are

$$
\lambda_{1}=-\sqrt{-\hat{p}_{v}(v, s)}, \quad \lambda_{2}=0, \quad \lambda_{3}=\sqrt{-\hat{p}_{v}(v, s)},
$$

and the sound speed $c(v, s)$ is defined by

$$
c(v, s)=v \sqrt{-\hat{p}_{v}(v, s)} .
$$

We divide the half space $(v, u, \theta), u>0$ into three regions:

$$
\begin{aligned}
& \Omega_{\text {sub }}=\{(v, u, \theta) \mid u<c(v, s), v>0, u>0, \theta>0\}, \\
& \Gamma_{\text {trans }}=\{(v, u, \theta) \mid u=c(v, s), v>0, u>0, \theta>0\}, \\
& \Omega_{\text {super }}=\{(v, u, \theta) \mid u>c(v, s), v>0, u>0, \theta>0\} .
\end{aligned}
$$

We call them the subsonic, transonic, and supersonic regions, respectively. For the inflow problem, we mainly consider the situation when $\left(v_{+}, u_{+}, \theta_{+}\right) \in \Omega_{s u b}$. If the left state $\left(v_{-}, u_{-}, \theta_{-}\right)$is sufficiently close to $\left(v_{+}, u_{+}, \theta_{+}\right)$such that $\left(v_{-}, u_{-}, \theta_{-}\right) \in \Omega_{\text {sub }}$ also 
holds, then the first characteristic speed $\lambda_{1}\left(v_{-}, u_{-}, \theta_{-}\right)$is less than $k_{-}$due to (1.13). Thus a travelling wave solution

$$
\begin{aligned}
& \left(V_{B}, U_{B}, \Theta_{B}\right)(\xi), \xi=x-k_{-} t \\
& \left(V_{B}, U_{B}, \Theta_{B}\right)(0)=\left(v_{-}, u_{-}, \theta_{-}\right), \quad\left(V_{B}, U_{B}, \Theta_{B}\right)(+\infty)=\left(v_{+}, u_{+}, \theta_{+}\right)
\end{aligned}
$$

is expected. Substituting this into (1.6), we have

$$
\left\{\begin{array}{l}
-k_{-} V_{B}^{\prime}-U_{B}^{\prime}=0, \quad '=\frac{d}{d \xi}, \quad \xi=x-k_{-} t>0 \\
-k_{-} U_{B}^{\prime}+p\left(V_{B}, \Theta_{B}\right)^{\prime}=\mu\left(\frac{U_{B}^{\prime}}{V_{B}}\right)^{\prime}, \\
-k_{-}\left(e\left(V_{B}, \Theta_{B}\right)+\frac{U_{B}^{2}}{2}\right)^{\prime}+\left(p\left(V_{B}, \Theta_{B}\right) U_{B}\right)^{\prime} \\
=\left(\kappa \frac{\Theta_{B}^{\prime}}{V_{B}}+\mu \frac{U_{B} U_{B}^{\prime}}{V_{B}}\right)^{\prime}, \\
\left(V_{B}, U_{B}, \Theta_{B}\right)(0)=\left(v_{-}, u_{-}, \theta_{-}\right), \\
\left(V_{B}, U_{B}, \Theta_{B}\right)(+\infty)=\left(v_{+}, u_{+}, \theta_{+}\right) .
\end{array}\right.
$$

We call the solution $\left(V_{B}, U_{B}, \Theta_{B}\right)$ the boundary layer solution, or BL-solution simply. If $\left(V_{B}, U_{B}, \Theta_{B}\right)$ exists, integration of $(1.14)$ over $(\xi,+\infty)$ yields

$$
\left\{\begin{array}{l}
-k_{-}\left(V_{B}-v_{+}\right)-\left(U_{B}-u_{+}\right)=0, \\
-k_{-}\left(U_{B}-u_{+}\right)+\left(p\left(V_{B}, \Theta_{B}\right)-p\left(v_{+}, \theta_{+}\right)\right)=\mu \frac{U_{B}^{\prime}}{V_{B}}, \\
-k_{-}\left(e\left(V_{B}, \Theta_{B}\right)-e\left(v_{+}, \theta_{+}\right)+\frac{U_{B}^{2}-u_{+}^{2}}{2}\right) \\
+\left(p\left(V_{B}, \Theta_{B}\right) U_{B}-p\left(v_{+}, \theta_{+}\right) u_{+}\right)=\left(\kappa \frac{\Theta_{B}^{\prime}}{V_{B}}+\mu \frac{U_{B} U_{B}^{\prime}}{V_{B}}\right) .
\end{array}\right.
$$

Let $\xi=0$ in $(1.15)$, then the first equation yields

$$
k_{-}=-\frac{U_{B}(\xi)}{V_{B}(\xi)}=-\frac{u_{-}}{v_{-}}=-\frac{u_{+}}{v_{+}} .
$$

Substituting (1.16) into $(1.15)_{2}$ and $(1.15)_{3}$, we have

$$
\left\{\begin{aligned}
V_{B}^{\prime}= & -\frac{V_{B}}{\mu k_{-}}\left[k_{-}^{2}\left(V_{B}-v_{+}\right)+\left(p\left(V_{B}, \Theta_{B}\right)-p\left(v_{+}, \theta_{+}\right)\right)\right] \\
:= & f\left(V_{B}, \Theta_{B}\right) \\
\Theta_{B}^{\prime}= & \frac{V_{B}}{\kappa}\left[-k_{-}\left(e\left(V_{B}, \Theta_{B}\right)-e\left(v_{+}, \theta_{+}\right)\right)\right. \\
& \left.-k_{-} p\left(v_{+}, \theta_{+}\right)\left(V_{B}-v_{+}\right)+\frac{k_{-}^{3}}{2}\left(V_{B}-v_{+}\right)^{2}\right] \\
:= & g\left(V_{B}, \Theta_{B}\right) .
\end{aligned}\right.
$$


Let

$$
\begin{aligned}
& W_{B}=\left(\begin{array}{c}
V_{B} \\
\Theta_{B}
\end{array}\right), F\left(W_{B}\right)=\left(\begin{array}{c}
f\left(V_{B}, \Theta_{B}\right) \\
g\left(V_{B}, \Theta_{B}\right)
\end{array}\right), \\
& W^{-}=\left(\begin{array}{c}
v_{-} \\
\theta_{-}
\end{array}\right), W^{+}=\left(\begin{array}{c}
v_{+} \\
\theta_{+}
\end{array}\right),
\end{aligned}
$$

and consider the system below

$$
\left\{\begin{array}{l}
W_{B}^{\prime}=F\left(W_{B}\right), F\left(W^{+}\right)=0, \\
W_{B}(0)=W^{-}, W_{B}(+\infty)=W^{+} .
\end{array}\right.
$$

The Jacobian matrix of (1.19) at $\xi=+\infty$ is

$$
J_{+}=\left(\begin{array}{cc}
-\frac{v_{+}}{\mu k_{-}}\left(k_{-}^{2}+p_{v}\left(v_{+}, \theta_{+}\right)\right) & -\frac{v_{+}}{\mu k_{-}} p_{\theta}\left(v_{+}, \theta_{+}\right) \\
-\frac{k_{-} v_{+}}{\kappa}\left(e_{v}\left(v_{+}, \theta_{+}\right)+p\left(v_{+}, \theta_{+}\right)\right) & -\frac{k_{-} v_{+}}{\kappa} e_{\theta}\left(v_{+}, \theta_{+}\right)
\end{array}\right) .
$$

Since $\left(v_{+}, u_{+}, \theta_{+}\right) \in \Omega_{\text {sub }},(1.9),(1.10)$, and (1.13) yield

$$
\begin{aligned}
\operatorname{det} J_{+} & =\left.\frac{v_{+}^{2}}{\mu \kappa}\left[\left(k_{-}^{2}+p_{v}\right) e_{\theta}-\left(e_{v}+p\right) p_{\theta}\right]\right|_{\left(v_{+}, \theta_{+}\right)} \\
& =\left.\frac{v_{+}^{2}}{\mu \kappa}\left[\left(k_{-}^{2}+\hat{p}_{v}\right) e_{\theta}\right]\right|_{\left(v_{+}, \theta_{+}\right)}<0 .
\end{aligned}
$$

Thus $J_{+}$has two eigenvalues $\Lambda_{1}<0<\Lambda_{2}$. On the contrary, by [5] and (1.16) there exists a local (unique) one dimensional stable manifold $M_{+}=M_{+}\left(v_{+}, u_{+}, \theta_{+}\right)$around $\left(v_{+}, u_{+}, \theta_{+}\right)$such that for any $\left(v_{-}, u_{-}, \theta_{-}\right) \in M_{+}$there exists a unique solution to (1.14) satisfying

$$
\begin{aligned}
& \left(\left|V_{B}-v_{+}\right|,\left|U_{B}-u_{+}\right|,\left|\Theta_{B}-\theta_{+}\right|\right)(\xi)=O(\delta) e^{-c \xi} \\
& \left(\left|V_{B}^{\prime}\right|,\left|V_{B}^{\prime \prime}\right|,\left|U_{B}^{\prime}\right|,\left|U_{B}^{\prime \prime}\right|,\left|\Theta_{B}^{\prime}\right|,\left|\Theta_{B}^{\prime \prime}\right|\right)(\xi)=O(\delta) e^{-c \xi}
\end{aligned}
$$

where $\delta=\left|v_{-}-v_{+}\right|+\left|u_{-}-u_{+}\right|+\left|\theta_{-}-\theta_{+}\right|$and the positive constant $c$ is independent of $\delta$.

On the other hand, for any given right state $\left(v_{+}, u_{+}, \theta_{+}\right)$, the $i$-rarefaction wave curve $(i=1,3)$ through $\left(v_{+}, u_{+}, \theta_{+}\right)$is known as

$$
\begin{aligned}
& R_{1}\left(v_{+}, u_{+}, \theta_{+}\right)=\left\{(v, u, \theta) \mid s=s_{+}, u=u_{+}-\int_{v_{+}}^{v} \lambda_{1}\left(\eta, s_{+}\right) d \eta, v<v_{+}\right\}, \\
& R_{3}\left(v_{+}, u_{+}, \theta_{+}\right)=\left\{(v, u, \theta) \mid s=s_{+}, u=u_{+}-\int_{v_{+}}^{v} \lambda_{3}\left(\eta, s_{+}\right) d \eta, v>v_{+}\right\},
\end{aligned}
$$

where $s_{+}=s\left(v_{+}, \theta_{+}\right)$. When $\left(v_{+}, u_{+}, \theta_{+}\right) \in \Omega_{s u b}$, we define

$$
\begin{aligned}
& B L R_{3}\left(v_{+}, u_{+}, \theta_{+}\right)=\bigcup M_{+}\left(v_{*}, u_{*}, \theta_{*}\right), \\
& \left(v_{*}, u_{*}, \theta_{*}\right) \in R_{3}\left(v_{+}, u_{+}, \theta_{+}\right) \cap \Omega_{\text {sub }} .
\end{aligned}
$$


If $\left(v_{-}, u_{-}, \theta_{-}\right) \in B L R_{3}\left(v_{+}, u_{+}, \theta_{+}\right)$, there exists a unique point $\left(v_{*}, u_{*}, \theta_{*}\right) \in \Omega_{\text {sub }}$ such that $\left(v_{*}, u_{*}, \theta_{*}\right) \in R_{3}\left(v_{+}, u_{+}, \theta_{+}\right)$and $\left(v_{-}, u_{-}, \theta_{-}\right) \in M_{+}\left(v_{*}, u_{*}, \theta_{*}\right)$. In fact, by (1.16), we have $u_{*}=-k_{-} v_{*}$. Substituting into (1.25) yields that

$$
-k_{-} v_{*}=u_{+}-\int_{v_{+}}^{v_{*}} \lambda_{3}\left(\eta, s_{+}\right) d \eta
$$

To the contrary, it is easy to see that $v_{*}$ is uniquely determined by (1.27) due to the fact that $-k_{-} v$ is increasing and the right hand side is decreasing with respect to $v>v_{+}$. After $v_{*}$ is obtained, we obtain $\theta_{*}=\theta\left(v_{*}, s_{+}\right)$.

When $\left(v_{-}, u_{-}, \theta_{-}\right) \in \Omega_{\text {super }}$, then $0>\lambda\left(v_{-}, u_{-}, \theta_{-}\right)>k_{-}$. Thus the behavior of the solutions is expected to be the same as that for the Cauchy problem. We define

$$
R_{1} R_{3}\left(v_{+}, u_{+}, \theta_{+}\right)=\bigcup R_{1}\left(v_{*}, u_{*}, \theta_{*}\right), \quad\left(v_{*}, u_{*}, \theta_{*}\right) \in R_{3}\left(v_{+}, u_{+}, \theta_{+}\right) .
$$

Our aim is to investigate the stability of the rarefaction wave, the BL-solution and their combination for the impermeable wall problem and inflow problem. Our results are, roughly speaking, as follows:

\section{(1) Impermeable problem:}

For any given right state $\left(v_{+}, u_{+}, \theta_{+}\right)$with $u_{+}>0$, there exist unique $v_{-}$and $\theta_{-}$ such that $\left(v_{-}, 0, \theta_{-}\right) \in R_{3}\left(v_{+}, u_{+}, \theta_{+}\right)$and the 3 -rarefaction wave connecting $\left(v_{-}, 0, \theta_{-}\right)$ and $\left(v_{+}, u_{+}, \theta_{+}\right)$is stable provided that $\left|v_{+}-v_{-}\right|+u_{+}+\left|\theta_{+}-\theta_{-}\right|$is small.

\section{(2) Inflow problem:}

(I) For any $\left(v_{+}, u_{+}, \theta_{+}\right) \in \Omega_{s u b}$, if $\left(v_{-}, u_{-}, \theta_{-}\right) \in M_{+}\left(v_{+}, u_{+}, \theta_{+}\right)$, then the BLsolution, connecting $\left(v_{-}, u_{-}, \theta_{-}\right)$and $\left(v_{+}, u_{+}, \theta_{+}\right)$is stable provided that $\left|v_{+}-v_{-}\right|+$ $\left|u_{+}-u_{-}\right|+\left|\theta_{+}-\theta_{-}\right|$is small.

(II) For any $\left(v_{+}, u_{+}, \theta_{+}\right) \in \Omega_{s u b}$, if $\left(v_{-}, u_{-}, \theta_{-}\right) \in B L R_{3}\left(v_{+}, u_{+}, \theta_{+}\right)$then there exists a unique point $\left(v_{*}, u_{*}, \theta_{*}\right) \in R_{3}\left(v_{+}, u_{+}, \theta_{+}\right)$such that the superposition of the BLsolution connecting $\left(v_{-}, u_{-}, \theta_{-}\right)$with $\left(v_{*}, u_{*}, \theta_{*}\right)$ and the 3 -rarefaction wave connecting $\left(v_{*}, u_{*}, \theta_{*}\right)$ with $\left(v_{+}, u_{+}, \theta_{+}\right)$is stable provided that $\left|v_{*}-v_{-}\right|+\left|u_{*}-u_{-}\right|+\left|\theta_{*}-\theta_{-}\right|$and $\left|v_{+}-v_{*}\right|+\left|u_{+}-u_{*}\right|+\left|\theta_{+}-\theta_{*}\right|$ are small.

(III) If $\left(v_{-}, u_{-}, \theta_{-}\right) \in R_{1} R_{3}\left(v_{+}, u_{+}, \theta_{+}\right) \cap \Omega_{\text {super }}$, then there exists a unique point $\left(v_{*}, u_{*}, \theta_{*}\right) \in R_{3}\left(v_{+}, u_{+}, \theta_{+}\right)$such that the superposition of the 1-rarefaction wave connecting $\left(v_{-}, u_{-}, \theta_{-}\right)$with $\left(v_{*}, u_{*}, \theta_{*}\right)$ and the 3 -rarefaction wave connecting $\left(v_{*}, u_{*}, \theta_{*}\right)$ with $\left(v_{+}, u_{+}, \theta_{+}\right)$is stable provided that $\left|v_{*}-v_{-}\right|+\left|u_{*}-u_{-}\right|+\left|\theta_{*}-\theta_{-}\right|$and $\left|v_{+}-v_{*}\right|+$ $\left|u_{+}-u_{*}\right|+\left|\theta_{+}-\theta_{*}\right|$ are small.

Our plan of this paper is as follows. In section 2, we discuss the impermeable wall problem. In section 3 the inflow problem is treated.

Notations. Throughout this paper, several positive generic constants are denoted by $C$ without confusions. For function spaces, $H^{l}(\Omega)$ denotes the $l$-th order Sobolev space with its norm

$$
\|f\|_{l}=\left(\sum_{j=0}^{l}\left\|\partial_{x}^{j} f\right\|^{2}\right)^{\frac{1}{2}}, \quad \text { when }\|\cdot\|:=\|\cdot\|_{L^{2}(\Omega)}
$$

The domain $\Omega$ will be often abbreviated without confusion. 


\section{Impermeable wall problem}

For any right state $\left(v_{+}, u_{+}, \theta_{+}\right)$with $u_{+}>0$, it is obvious that there exist unique $v_{-}>0$ and $\theta_{-}>0$ such that $\left(v_{-}, 0, \theta_{-}\right) \in R_{3}\left(v_{+}, u_{+}, \theta_{+}\right)$. The 3 -rarefaction wave $\left(v^{r}, u^{r}, \theta^{r}\right)\left(\frac{x}{t}\right)$ connecting $\left(v_{-}, 0, \theta_{-}\right)$and $\left(v_{+}, u_{+}, \theta_{+}\right)$is the weak solution of the Riemann problem

$$
\left\{\begin{array}{l}
v_{t}-u_{x}=0, \\
u_{t}+p(v, \theta)_{x}=0, \\
\left(e(v, \theta)+\frac{u^{2}}{2}\right)_{t}+(p(v, \theta) u)_{x}=0, \\
\left(v_{0}, u_{0}, \theta_{0}\right)(x)=\left\{\begin{array}{l}
\left(v_{-}, 0, \theta_{-}\right), x<0, \\
\left(v_{+}, u_{+}, \theta_{+}\right), x>0 .
\end{array}\right.
\end{array}\right.
$$

To study the large time behavior of the solutions, it is necessary to construct a smooth approximate rarefaction wave $(V, U, \Theta)(x, t)$ of $\left(v^{r}, u^{r}, \theta^{r}\right)\left(\frac{x}{t}\right)$ in $\mathbb{R}_{+} \times(0,+\infty)$. To this end, we apply the idea of [1]. We first construct the solution $w(x, t)$ of the following problem

$$
\left\{\begin{array}{l}
w_{t}+w w_{x}=0, \quad(x, t) \in \mathbb{R} \times(0,+\infty), \\
\left.w\right|_{t=0}= \begin{cases}w_{-}, & x<0 \\
w_{-}+\tilde{w} K_{q} \int_{0}^{\varepsilon x} z^{q} e^{-z} d z, & x \geq 0\end{cases}
\end{array}\right.
$$

where $w_{ \pm}=\lambda_{3}\left(v_{ \pm}, s_{+}\right), s_{+}=s\left(v_{+}, \theta_{+}\right), \tilde{w}=w_{+}-w_{-}, K_{q}$ is a constant such that $K_{q} \int_{0}^{+\infty} z^{q} e^{-z} d z=1$ for large constant $q \geq 8$ and a small positive constant $\varepsilon$. We have the following properties of $w(x, t)$ due to [1].

LEMma 2.1. [1] Let $0<w_{-}<w_{+}$. Then the problem (2.2) has a unique smooth solution $w(x, t)$ satisfying

i. $w_{-} \leq w(x, t)<w_{+}, w_{x} \geq 0$, for $x \geq 0, t \geq 0$.

ii. For any $p(1 \leq p \leq+\infty)$, there exists a constant $C_{p, q}$ such that for $t \geq 0$,

$$
\begin{aligned}
& \left\|w_{x}(\cdot, t)\right\|_{L^{p}} \leq C_{p, q} \min \left(\tilde{w} \varepsilon^{1-\frac{1}{p}}, \tilde{w}^{\frac{1}{p}} t^{-1+\frac{1}{p}}\right), \\
& \left\|w_{x x}(\cdot, t)\right\|_{L^{p}} \leq C_{p, q} \min \left(\tilde{w} \varepsilon^{2-\frac{1}{p}}, \tilde{w}^{\frac{1}{q}} \varepsilon^{1-\frac{1}{p}+\frac{1}{q}} t^{-1+\frac{1}{q}}\right) .
\end{aligned}
$$

iii. when $x \leq 0, w(x, t)-w_{-}=w_{x}(x, t)=w_{x x}(x, t)=0$.

iv. $\limsup _{t \rightarrow+\infty, x \in \Re}\left|w(x, t)-w^{R}(x, t)\right|=0$.

Here $w^{R}(x, t)$ is the Riemann solution of the scalar equation (2.2) with the initial data $w_{0}(x)=w_{-}$, if $x<0$, and $w_{0}(x)=w_{+}$, if $x>0$.

Then the smooth approximation $\left(V^{r}, U^{r}, \Theta^{r}\right)$ to $\left(v^{r}, u^{r}, \theta^{r}\right)(x, t)$ is given by

$$
\left\{\begin{array}{l}
S^{r}(x, t)=s_{+}, \\
\lambda_{3}\left(V^{r}(x, t), s_{+}\right)=w(x, t), \\
U^{r}(x, t)=u_{+}-\int_{v_{+}}^{V^{r}(x, t)} \lambda_{3}\left(\eta, s_{+}\right) d \eta .
\end{array}\right.
$$

Setting

$$
(V, U, \Theta)(x, t):=\left.\left(V^{r}, U^{r}, \Theta^{r}\right)(x, t)\right|_{x \geq 0},
$$


then one easily has

$$
\begin{cases}V_{t}-U_{x}=0, & x>0, t>0, \\ U_{t}+p(V, \Theta)_{x}=0, & x>0, t>0, \\ \left(e(V, \Theta)+\frac{1}{2} U^{2}\right)_{t}+(p(V, \Theta) U)_{x}=0, & x>0, t>0, \\ \left.(V, U, \Theta)\right|_{x=0}=\left(v_{-}, 0, \theta_{-}\right), & \\ \left.(V, U, \Theta)\right|_{t=0}=\left(V_{0}, U_{0}, \Theta_{0}\right)(x)=(V, U, \Theta)(x, 0) .\end{cases}
$$

Due to Lemma 2.1, $(V, U, \Theta)$ has the following properties.

Lemma 2.2. Let $\delta_{1}=\left|v_{+}-v_{-}\right|+u_{+}+\left|\theta_{+}-\theta_{-}\right|$. Then $(V, U, \Theta)(x, t)$ satisfies, if $q \geq p$,

i. $\quad U_{x}(x, t) \geq 0,\left|U_{x}\right| \leq C \varepsilon \delta_{1}$, for $t \geq 0, x \geq 0$,

ii. For any $p(1 \leq p \leq+\infty)$, there exists a constant $C_{p, q}$ such that

$$
\begin{aligned}
& \left\|\left(V_{x}, U_{x}, \Theta_{x}\right)\right\|_{L^{p}(x \geq 0)} \leq C_{p, q} \min \left\{\delta_{1} \varepsilon^{1-\frac{1}{p}}, \delta_{1}^{\frac{1}{p}}(1+t)^{-1+\frac{1}{p}}\right\}, \\
& \left\|\left(V_{x x}, U_{x x}, \Theta_{x x}\right)\right\|_{L^{p}(x \geq 0)} \leq C_{p, q} \min \left\{\delta_{1} \varepsilon^{2-\frac{1}{p}}, \delta_{1}^{\frac{1}{q}}(1+t)^{-1+\frac{1}{q}}\right\}, \quad t \geq 0 .
\end{aligned}
$$

iii. $\left.\quad(V, U, \Theta)\right|_{x \leq 0}=\left(v_{-}, 0, \theta_{-}\right),\left.\quad\left(V_{x}, U_{x}, \Theta_{x}, V_{x x}, U_{x x}, \Theta_{x x}\right)\right|_{x \leq 0}=0$.

iv. $\limsup _{t \rightarrow+\infty, x \in\{x \geq 0\}}\left|(V, U, \Theta)(x, t)-\left(v^{r}, u^{r}, \theta^{r}\right)\left(\frac{x}{t}\right)\right|=0$.

We assume

$$
\left(v_{0}-V_{0}, u_{0}-U_{0}, \theta_{0}-\Theta_{0}\right) \in L^{2}\left(\mathbb{R}_{+}\right), \quad\left(v_{0}, u_{0}, \theta_{0}\right)_{x} \in L^{2}\left(\mathbb{R}_{+}\right),
$$

and set

$$
N_{0}=\left\|v_{0}-V_{0}\right\|_{1}+\left\|u_{0}-U_{0}\right\|_{1}+\left\|\theta_{0}-\Theta_{0}\right\|_{1} .
$$

It is noted that on the boundary $x=0,(V, U, \Theta)(0, t)=\left(v_{-}, 0, \theta_{-}\right)$and $(u-U, \theta-$ $\Theta)(0, t)=(0,0)$. That is, the boundary effect is exactly eliminated by our approximation because $(v-V)(0, t)$ is not important in the energy estimates. Thus the impermeable wall problem is similar to the Cauchy problem for the rarefaction wave case. Using the same lines as in [4], we have

THEOREM 2.3. For any right state $\left(v_{+}, u_{+}, \theta_{+}\right)$with $u_{+}>0$, there exist unique $v_{-}$and $\theta_{-}$such that $\left(v_{-}, 0, \theta_{-}\right) \in R_{3}\left(v_{+}, u_{+}, \theta_{+}\right)$. Suppose that the Assumption (2.6) holds. Then there exists a positive constant $\delta_{0}$ such that if $N_{0}+\delta_{1}<\delta_{0}$, the impermeable wall problem (1.6), (1.7) $)_{1}$, and (1.8) has a unique global solution in time satisfying

$$
\left\{\begin{array}{l}
(v-V, u-U, \theta-\Theta) \in C^{0}\left(0,+\infty ; H^{1}\right) \\
(v, u, \theta)_{x} \in L^{2}\left(0,+\infty ; L^{2}\right) \\
(u, \theta)_{x x} \in L^{2}\left(0,+\infty ; L^{2}\right)
\end{array}\right.
$$

and

$$
\limsup _{t \rightarrow+\infty, x>0}\left|(v, u, \theta)(x, t)-\left(v^{r}, u^{r}, \theta^{r}\right)\left(\frac{x}{t}\right)\right| \rightarrow 0 .
$$




\section{Inflow problem}

3.1. BL-solution. Assume that $\left(v_{+}, u_{+}, \theta_{+}\right) \in \Omega_{\text {sub }}$. Then there exists a local (unique) stable manifold $M_{+}=M_{+}\left(v_{+}, u_{+}, \theta_{+}\right)$such that for any $\left(v_{-}, u_{-}, \theta_{-}\right) \in M_{+}$, there exists a unique travelling wave solution $\left(V_{B}, U_{B}, \Theta_{B}\right)(\xi), \xi=x-k_{-} t$, satisfying (1.14). For simplicity, let $(V, U, \Theta)(\xi)=\left(V_{B}, U_{B}, \Theta_{B}\right)(\xi)$. We define the perturbation by

$$
(\phi, \psi, \zeta)(\xi, t)=(v, u, \theta)(\xi, t)-(V, U, \Theta)(\xi) .
$$

On the other hand, by (1.9) and (1.10) the third equation of (1.6) can be reduced to

$$
e_{\theta} \theta_{t}+\theta p_{\theta} u_{x}=\theta s_{t}=\kappa\left(\frac{\theta_{x}}{v}\right)_{x}+\mu \frac{u_{x}^{2}}{v} .
$$

Thus $(\phi, \psi, \zeta)$ satisfies,

$$
\left\{\begin{array}{l}
\phi_{t}-k_{-} \phi_{\xi}-\psi_{\xi}=0 \\
\psi_{t}-k_{-} \psi_{\xi}+[p(v, \theta)-p(V, \Theta)]_{\xi}=\mu\left(\frac{u_{\xi}}{v}\right)_{\xi}-\mu\left(\frac{U_{\xi}}{V}\right)_{\xi} \\
\zeta_{t}-k_{-} \zeta_{\xi}+\frac{\theta p_{\theta}}{e_{\theta}}(v, \theta) \psi_{\xi}+\left\{\frac{\theta p_{\theta}}{e_{\theta}}(v, \theta)-\frac{\Theta p_{\theta}}{e_{\theta}}(V, \Theta)\right\} U_{\xi} \\
=\frac{1}{e_{\theta}(v, \theta)}\left\{\left(\frac{\kappa \theta_{\xi}}{v}\right)_{\xi}+\frac{\mu u_{\xi}^{2}}{v}\right\}-\frac{1}{e_{\theta}(V, \Theta)}\left\{\left(\frac{\kappa \Theta \xi}{V}\right)_{\xi}+\frac{\mu U_{\xi}^{2}}{V}\right\} \\
\left.(\phi, \psi, \zeta)\right|_{\xi=0}=0, \\
\left.(\phi, \psi, \zeta)\right|_{t=0}=\left(\phi_{0}, \psi_{0}, \zeta_{0}\right)=\left(v_{0}(\xi)-V, u_{0}-U, \theta_{0}-\Theta\right)(\xi)
\end{array}\right.
$$

We have the following stability theorem for the BL-solution.

THEOREM 3.1. Suppose that $\left(\phi_{0}, \psi_{0}, \zeta_{0}\right) \in H_{0}^{1}\left(R_{+}\right)$. Then there exists a positive constant $\delta_{0}$ such that if

$$
\left\|\left(\phi_{0}, \psi_{0}, \zeta_{0}\right)\right\|_{1}+\left|v_{+}-v_{-}\right|+\left|u_{+}-u_{-}\right|+\left|\theta_{+}-\theta_{-}\right| \leq \delta_{0},
$$

then the initial boundary value problem (3.3) has a unique global solution in time satisfying

$$
\left\{\begin{array}{l}
(\phi, \psi, \zeta) \in C^{0}\left(0,+\infty ; H_{0}^{1}\right) \\
\phi_{\xi} \in L^{2}\left(0,+\infty ; L^{2}\right) \\
(\psi, \zeta)_{\xi} \in L^{2}\left(0,+\infty ; H^{1}\right)
\end{array}\right.
$$

and

$$
\limsup _{t \rightarrow+\infty, \xi>0}|(\phi, \psi, \zeta)(\xi, t)| \rightarrow 0
$$

To show the stability Theorem 3.1, we seek the solution $(\phi, \psi, \zeta)$ in the solution space

$$
\begin{aligned}
& X(0, T)=\left\{(\phi, \psi, \zeta) \in C^{0}\left(0, T ; H_{0}^{1}\right) \mid \phi_{\xi} \in L^{2}\left(0, T ; L^{2}\right)\right. \\
& \left.\left(\psi_{\xi}, \zeta_{\xi}\right) \in L^{2}\left(0, T ; H^{1}\right), \sup _{[0, T]}\|(\phi, \psi, \zeta)(t)\|_{1} \leq \varepsilon_{1}\right\}
\end{aligned}
$$


where $\varepsilon_{1}=\frac{1}{4} \min \left\{v_{-}, v_{+}\right\}$. Let $\delta=\left|v_{+}-v_{-}\right|+\left|u_{+}-u_{-}\right|+\left|\theta_{+}-\theta_{-}\right|$and

$$
N(T)=\sup _{0 \leq \tau \leq T}\left(\|\phi(\tau)\|_{1}+\|\psi(\tau)\|_{1}+\|\zeta(\tau)\|_{1}\right) .
$$

Then $N(T) \leq \varepsilon_{1}$ which ensures the system (3.3) is nonsingular. By using the same lines as in the previous papers [12-13], it is easy to prove the local existence. We omit the proofs here. To justify Theorem 3.1, it is necessary to establish the a priori estimates. We have

Proposition 3.2. There exist positive constants $\delta_{0}, \varepsilon_{2}\left(\leq \varepsilon_{1}\right)$ and $C_{0}$ such that, if $\delta_{1} \leq \delta_{0}, N(T) \leq \varepsilon_{2}$ and $(\phi, \psi, \zeta)(\xi, t) \in X(0, T)$ is a solution of (3.3) for some $T>0$, then $(\phi, \psi, \zeta)$ satisfies

$$
\begin{aligned}
& \|(\phi, \psi, \zeta)(t)\|_{1}^{2}+\int_{0}^{t}\left\{\left\|\phi_{\xi}(\tau)\right\|^{2}+\left\|(\psi, \zeta)_{\xi}(\tau)\right\|_{1}^{2}\right\} d \tau \\
\leq & C_{0}\|(\phi, \psi, \zeta)(0)\|^{2} .
\end{aligned}
$$

Proposition 3.2 is proved by a series lemmas. We first give the following key lemma.

LEMMA 3.3. It follows that

$$
\begin{aligned}
& \|(\phi, \psi, \zeta)(t)\|^{2}+\int_{0}^{t}\left\{\left\|\psi_{\xi}(\tau)\right\|^{2}+\left\|\zeta_{\xi}(\tau)\right\|^{2}\right\} d \tau \\
\leq & C\|(\phi, \psi, \zeta)(0)\|^{2}+C \delta \int_{0}^{t}\left\|\phi_{\xi}\right\|^{2}(\tau) d \tau .
\end{aligned}
$$

Proof. Let

$$
\mathcal{E}=e(v, s)-e(V, S)+\frac{\psi^{2}}{2}+p(V, \Theta)(v-V)-\Theta(s-S) .
$$

Then by (1.9)-(1.10), (3.2)-(3.3), we have

$$
\begin{aligned}
& \mathcal{E}_{t}+\frac{\mu \psi_{\xi}^{2}}{v}+\frac{\kappa \zeta_{\xi}^{2}}{v \theta}+\Delta_{1} U_{\xi}+k_{-} \Delta_{2} S_{\xi} \\
= & \left(k_{-} \mathcal{E}+\mu \psi\left(\frac{u_{\xi}}{v}-\frac{U_{\xi}}{V}\right)+\kappa \zeta\left(\frac{\theta_{\xi}}{v \theta}-\frac{\Theta_{\xi}}{V \Theta}\right)-(p(v, \theta)-p(V, \Theta)) \psi\right)_{\xi} \\
& +\frac{\mu \phi \psi_{\xi} U_{\xi}}{v V}+\mu \zeta\left(\frac{u_{\xi}^{2}}{v \theta}-\frac{U_{\xi}^{2}}{V \Theta}\right)+\kappa \zeta\left(\frac{\theta_{\xi}^{2}}{v \theta^{2}}-\frac{\Theta_{\xi}^{2}}{V \Theta^{2}}\right)-\kappa \zeta_{\xi} \Theta_{\xi}\left(\frac{1}{v \theta}-\frac{1}{V \Theta}\right),
\end{aligned}
$$

where

$$
\begin{aligned}
& \Delta_{1}=\hat{p}(v, s)-\hat{p}(V, S)-\hat{p}_{v}(V, S) \phi-\hat{p}_{s}(V, S)(s-S), \\
& \Delta_{2}=\theta-\Theta-\hat{\theta}_{v}(V, S) \phi-\hat{\theta}_{s}(V, S)(s-S) .
\end{aligned}
$$

satisfy $\left|\Delta_{i}\right| \leq C\left(\phi^{2}+\zeta^{2}\right), i=1,2$. It is noted that $e(v, s)$ is a strictly convex function of $v$ and $s$ due to (1.2), (1.9), and (1.10). Since $s_{\theta}=\frac{e_{\theta}}{\theta}>0$, the Cauchy inequality yields that there exists a positive constant $c_{1}>0$ such that

$$
\mathcal{E} \geq c_{1}\left(\phi^{2}+\psi^{2}+\zeta^{2}\right) .
$$


Note that $(\phi, \psi, \zeta)(0, t)=(0,0,0)$. Integrating $(3.12)$ over $[0, t] \times \mathbb{R}_{+}$, we have

$$
\begin{aligned}
& \|(\phi, \psi, \zeta)(t)\|^{2}+\int_{0}^{t}\left\|\partial_{\xi}(\psi, \zeta)(\tau)\right\|^{2} d \tau \\
\leq & C_{1}\|(\phi, \psi, \zeta)(0)\|^{2}+C_{1}\left|\int_{0}^{t} \int_{0}^{+\infty} \frac{\mu \phi \psi_{\xi} U_{\xi}}{v V}+\mu \zeta\left(\frac{u_{\xi}^{2}}{v \theta}-\frac{U_{\xi}^{2}}{V \Theta}\right)-\Delta_{1} U_{\xi} d \xi d t\right| \\
& +C_{1}\left|\int_{0}^{t} \int_{0}^{+\infty} \kappa \zeta\left(\frac{\theta_{\xi}^{2}}{v \theta^{2}}-\frac{\Theta_{\xi}^{2}}{V \Theta^{2}}\right)-\kappa \zeta_{\xi} \Theta_{\xi}\left(\frac{1}{v \theta}-\frac{1}{V \Theta}\right)-k_{-} \Delta_{2} S_{\xi} d \xi d t\right| \\
= & : C_{1}\|(\phi, \psi, \zeta)(0)\|^{2}+I_{1}+I_{2} .
\end{aligned}
$$

We use the idea of [6] to estimate the right hand side of (3.14). Since

$$
\left|\left(\phi^{2}, \psi^{2}, \zeta^{2}\right)(\xi)\right| \leq \xi\left\|\left(\phi_{\xi}, \psi_{\xi}, \zeta_{\xi}\right)\right\|^{2}, \forall \xi>0,
$$

the Cauchy inequality and (1.23) yield that

$$
I_{1} \leq C \delta \int_{0}^{t}\left\|\left(\phi_{\xi}, \psi_{\xi}, \zeta_{\xi}\right)\right\|^{2} d t+C N(T) \int_{0}^{t}\left\|\psi_{\xi}\right\|^{2} d t
$$

and

$$
I_{2} \leq C \delta \int_{0}^{t}\left\|\left(\phi_{\xi}, \psi_{\xi}, \zeta_{\xi}\right)\right\|^{2} d t+C N(T) \int_{0}^{t}\left\|\zeta_{\xi}\right\|^{2} d t .
$$

Thus there exist positive small constants $\delta_{0}$ and $\varepsilon_{2}$. When $\delta \leq \delta_{0}$ and $N(T) \leq \varepsilon_{2}$, substituting (3.16)-(3.17) into (3.14) yields the desired estimate (3.10).

Lemma 3.4. It follows that

$$
\left\|\phi_{\xi}(t)\right\|^{2}+\int_{0}^{t}\left\|\phi_{\xi}\right\|^{2} d \tau \leq C\|(\phi, \psi, \zeta)(0)\|_{1}^{2}+C \int_{0}^{t} \check{v}_{\xi}^{2}(0, t) d t .
$$

Proof. Following [11], we introduce a new variable $\check{v}=\frac{v}{V}$. Then $(3.3)_{2}$ is rewritten as

$$
\left(\mu \frac{\check{v}_{\xi}}{\check{v}}-\psi\right)_{t}=k_{-}\left(\mu \frac{\check{v}_{\xi}}{\check{v}}-\psi\right)_{\xi}+(p(v, \theta)-p(V, \Theta))_{\xi} .
$$

Multiplying (3.19) by $\frac{\check{v}_{\xi}}{\grave{v}}$, we have

$$
\begin{aligned}
& \left\{\frac{\mu}{2}\left(\frac{\check{v}_{\xi}}{\check{v}}\right)^{2}-\psi \frac{\check{v}_{\xi}}{\check{v}}\right\}_{t}-p_{v}(v, \theta) V \frac{\check{v}_{\xi}^{2}}{\check{v}}+\left(\frac{\check{v}_{t}}{\check{v}} \psi-\frac{k_{-} \mu}{2}\left(\frac{\check{v}_{\xi}}{\check{v}}\right)^{2}\right)_{\xi} \\
& =\mu \frac{\psi_{\xi}^{2}}{v}-\frac{\mu \phi \psi_{\xi} U_{\xi}}{v V}+\left(p_{v}(v, \theta) \check{v}-p_{v}(V, \Theta)\right) V_{\xi} \frac{\check{v}_{\xi}}{\check{v}} \\
& +\left(p_{\theta}(v, \theta) \theta_{\xi}-p_{\theta}(V, \Theta) \Theta_{\xi}\right) \frac{\check{v}_{\xi}}{\check{v}} .
\end{aligned}
$$

We compute

$$
\left|\left(p_{v}(v, \theta) \check{v}-p_{v}(V, \Theta)\right) V_{\xi} \frac{\check{v}_{\xi}}{\check{v}}\right| \leq \tilde{\delta} \check{v}_{\xi}^{2}+C_{\tilde{\delta}}\left(\phi^{2}+\zeta^{2}\right) V_{\xi}^{2},
$$




$$
\begin{gathered}
\left|\left(p_{\theta}(v, \theta) \theta_{\xi}-p_{\theta}(V, \Theta) \Theta_{\xi}\right) \frac{\check{v}_{\xi}}{\check{v}}\right| \leq \tilde{\delta} \tilde{v}_{\xi}^{2}+C_{\tilde{\delta}} \zeta_{\xi}^{2}+C_{\tilde{\delta}}\left(\phi^{2}+\zeta^{2}\right) \Theta_{\xi}^{2}, \\
\left|\frac{\mu \phi \psi_{\xi} U_{\xi}}{v V}\right| \leq C \phi^{2} U_{\xi}^{2}+C \psi_{\xi}^{2}, \\
\int_{0}^{\infty}\left(\phi^{2}+\zeta^{2}\right)\left(V_{\xi}^{2}+U_{\xi}^{2}+\Theta_{\xi}^{2}\right) d x \leq C \delta^{2}\left(\left\|\phi_{\xi}\right\|^{2}+\left\|\zeta_{\xi}\right\|^{2}\right),
\end{gathered}
$$

and

$$
c_{2} \phi_{\xi}^{2}-c_{3} \phi^{2} V_{\xi}^{2} \leq \check{v}_{\xi}^{2} \leq C_{2} \phi_{\xi}^{2}+C_{3} \phi^{2} V_{\xi}^{2},
$$

where $c_{i}, C_{i}, i=2,3$ are positive constants which only depend on $v_{-}$and $v_{+}$. Note that $\delta$ is small. Thus integrating (3.20) on $R_{+} \times[0, t]$, using (3.21)-(3.25) and Lemma 3.3 and choosing $\tilde{\delta}$ is suitably small, we get (3.18). Thus Lemma 3.4 is proved.

LEMma 3.5. It follows that

$$
\left\|\left(\psi_{\xi}, \zeta_{\xi}\right)(t)\right\|^{2}+\int_{0}^{t}\left\|\left(\psi_{\xi \xi}, \zeta_{\xi \xi}\right)(\tau)\right\|^{2} d \tau \leq C\|(\phi, \psi, \zeta)(0)\|_{1}^{2} .
$$

Proof. $\quad$ Multiplying $(3.3)_{2}$ by $-\psi_{\xi \xi}$ and $(3.3)_{3}$ by $-\zeta_{\xi \xi}$, and adding the resulting equalities, we have

$$
\begin{aligned}
& \left(\frac{1}{2} \psi_{\xi}^{2}+\frac{1}{2} \zeta_{\xi}^{2}\right)_{t}+\left(\frac{k_{-}}{2}\left(\psi_{\xi}^{2}+\zeta_{\xi}^{2}\right)-\psi_{t} \psi_{\xi}-\zeta_{t} \zeta_{\xi}\right)_{\xi}+\mu \frac{\psi_{\xi \xi}^{2}}{v}+\kappa \frac{\zeta_{\xi \xi}^{2}}{v e_{\theta}(v, \theta)} \\
= & (p(v, \theta)-p(V, \Theta))_{\xi} \psi_{\xi \xi}+\left(\frac{\theta p_{\theta}}{e_{\theta}}(v, \theta) u_{\xi}-\frac{\Theta p_{\theta}}{e_{\theta}}(V, \Theta) U_{\xi}\right) \zeta_{\xi \xi} \\
& -\mu \psi_{\xi \xi} U_{\xi \xi}\left(\frac{1}{v}-\frac{1}{V}\right)+\mu \psi_{\xi \xi}\left(\frac{u_{\xi} v_{\xi}}{v^{2}}-\frac{U_{\xi} V_{\xi}}{V^{2}}\right) \\
& -\kappa \zeta_{\xi \xi} \Theta_{\xi \xi}\left(\frac{1}{e_{\theta} v}(v, \theta)-\frac{1}{e_{\theta} V}(V, \Theta)\right)+\kappa \zeta_{\xi \xi}\left(\frac{\theta_{\xi} v_{\xi}}{e_{\theta} v^{2}}(v, \theta)-\frac{\Theta_{\xi} V_{\xi}}{e_{\theta} V^{2}}(V, \Theta)\right) \\
& -\mu \zeta_{\xi \xi}\left(\frac{u_{\xi}^{2}}{e_{\theta} v}(v, \theta)-\frac{U_{\xi}^{2}}{e_{\theta} V}(V, \Theta)\right) .
\end{aligned}
$$

We estimate

$$
\begin{aligned}
\mathrm{RHS} \leq & \tilde{\delta}\left(\psi_{\xi \xi}^{2}+\zeta_{\xi \xi}^{2}\right)+C_{\tilde{\delta}}\left(\phi_{\xi}^{2}+\psi_{\xi}^{2}+\zeta_{\xi}^{2}\right)+C_{\tilde{\delta}}\left(\phi^{2}+\zeta^{2}\right)\left(V_{\xi}^{2}+U_{\xi}^{2}\right. \\
& \left.+\Theta_{\xi}^{2}+U_{\xi \xi}^{2}+\Theta_{\xi \xi}^{2}\right)+C\left(\left|\psi_{\xi \xi} \psi_{\xi} \phi_{\xi}\right|+\left|\zeta_{\xi \xi} \zeta_{\xi} \phi_{\xi}\right|+\left|\zeta_{\xi \xi} \psi_{\xi}^{2}\right|\right) .
\end{aligned}
$$

It is noted that

$$
\int_{0}^{\infty}\left|\psi_{\xi \xi} \psi_{\xi} \phi_{\xi}\right| d \xi \leq C\left\|\psi_{\xi \xi}\right\|^{\frac{3}{2}}\left\|\psi_{\xi}\right\|^{\frac{1}{2}}\left\|\phi_{\xi}\right\| \leq C N(T)\left(\left\|\psi_{\xi}\right\|^{2}+\left\|\psi_{\xi \xi}\right\|^{2}\right) .
$$

The last two terms can be treated by the same way. Note that $k_{-}<0$ and $\psi_{t}(0, t)=$ $\zeta_{t}(0, t)=0$. Thus, integrating $(3.27)$ over $R_{+} \times[0, T]$, choosing $\tilde{\delta}$ suitably small and using Lemmas 3.3-3.4, we have

$$
\begin{aligned}
& \left\|\left(\phi_{\xi}, \psi_{\xi}, \zeta_{\xi}\right)(t)\right\|^{2}+\int_{0}^{t}\left(\left\|\phi_{\xi}(\tau)\right\|^{2}+\left\|\left(\psi_{\xi \xi}, \zeta_{\xi \xi}\right)(\tau)\right\|^{2}\right) d \tau \\
\leq & C\|(\phi, \psi, \zeta)(0)\|_{1}^{2}+C \int_{0}^{t}\left\|\psi_{\xi}\right\|^{2} d \tau,
\end{aligned}
$$


where we have used the fact that

$$
\check{v}_{\xi}^{2}(0, t) \leq C \phi_{\xi}^{2}(0, t) \leq C \psi_{\xi}^{2}(0, t) \leq \tilde{\delta}\left\|\psi_{\xi \xi}\right\|^{2}+C_{\tilde{\delta}}\left\|\psi_{\xi}\right\|^{2} .
$$

Adding (3.30) $\times \lambda$ and (3.10), we have

$$
\begin{aligned}
& \|(\phi, \psi, \zeta)(t)\|^{2}+\lambda\left\|\left(\phi_{\xi}, \psi_{\xi}, \zeta_{\xi}\right)(t)\right\|^{2}+(1-C \lambda) \int_{0}^{t}\left\|\psi_{\xi}\right\|^{2} d \tau \\
& +\int_{0}^{t}\left\|\zeta_{\xi}\right\|^{2} d \tau+(\lambda-C \delta) \int_{0}^{t}\left\|\phi_{\xi}\right\|^{2} d \tau+\lambda \int_{0}^{t}\left\|\left(\psi_{\xi \xi}, \zeta_{\xi \xi}\right)\right\|^{2} d \tau \\
\leq & C\|(\phi, \psi, \zeta)(0)\|_{1}^{2} .
\end{aligned}
$$

We now choose $\lambda=\frac{1}{2 C}>0$ such that $1-C \lambda=\frac{1}{2}$. Let $\delta$ be suitably small so that $\lambda-C \delta>\frac{1}{2} \lambda$. Therefore Lemma 3.5 is proved from (3.32).

Proof of Proposition 3.2. Proposition 3.2 is obtained at once from Lemmas 3.3-3.5.

3.2. The superposition of the BL-solution and the rarefaction wave.

In this section, we investigate the case $\left(v_{-}, u_{-}, \theta_{-}\right) \in B L R_{3}\left(v_{+}, u_{+}, \theta_{+}\right)$. According to the previous arguments, there exists a unique point $\left(v_{*}, u_{*}, \theta_{*}\right) \in \Omega_{\text {sub }}$ such that $\left(v_{*}, u_{*}, \theta_{*}\right) \in R_{3}\left(v_{+}, u_{+}, \theta_{+}\right)$. We study the stability of the superposition of the BL-solution $\left(V_{B}, U_{B}, \Theta_{B}\right)(\xi), \xi=x-k_{-} t$, connecting $\left(v_{-}, u_{-}, \theta_{-}\right)$with $\left(v_{*}, u_{*}, \theta_{*}\right)$ and the rarefaction wave $\left(v^{r}, u^{r}, \theta^{r}\right)\left(\frac{x}{t}\right)$ connecting $\left(v_{*}, u_{*}, \theta_{*}\right)$ with $\left(v_{+}, u_{+}, \theta_{+}\right)$. The BLsolution $\left(V_{B}, U_{B}, \Theta_{B}\right)$ satisfies (1.14), (1.22), and (1.23), where the right state is replaced by $\left(v_{*}, u_{*}, \theta_{*}\right)$ and $\delta=\left|v_{*}-v_{-}\right|+\left|u_{*}-u_{-}\right|+\left|\theta_{*}-\theta_{-}\right|$.

For the rarefaction wave, we use the same smooth approximation $\left(V^{r}, U^{r}, \Theta^{r}\right)$ to $\left(v^{r}, u^{r}, \theta^{r}\right)\left(\frac{x}{t}\right)$ as in $(2.2)$ and $(2.3)$, where the left state is replaced by $\left(v_{*}, u_{*}, \theta_{*}\right)$. Let

$$
\left(V_{3}, U_{3}, \Theta_{3}\right)(x, t):=\left.\left(V^{r}, U^{r}, \Theta^{r}\right)\right|_{x \geq k_{-} t} .
$$

If we introduce a new coordinate $t=t, \xi=x-k_{-} t$, then we have

$$
\begin{cases}V_{3 t}-k_{-} V_{3 \xi}-U_{\xi}=0, & \xi>0, t>0, \\ U_{3 t}-k_{-} U_{3 \xi}+p\left(V_{3}, \Theta_{3}\right)_{\xi}=0, & \xi>0, t>0, \\ \Theta_{3 t}-k_{-} \Theta_{3 \xi}+\frac{\Theta_{3} p_{\theta}}{e_{\theta}}\left(\Theta_{3}, V_{3}\right) U_{3 \xi}=0, & \xi>0, t>0, \\ \left.\left(V_{3}, U_{3}, \Theta_{3}\right)\right|_{\xi=0}=\left(v_{*}, u_{*}, \theta_{*}\right), & \left.\left(V_{3}, U_{3}, \Theta_{3}\right)\right|_{\xi \rightarrow+\infty}=\left(v_{+}, u_{+}, \theta_{+}\right), \\ \left.\left(V_{3}, U_{3}, \Theta_{3}\right)\right|_{t=0}=\left(V_{3}, U_{3}, \Theta_{3}\right)(\xi, 0) . & \end{cases}
$$

Similar to Lemma 2.2, $\left(V_{3}, U_{3}, \Theta_{3}\right)(\xi, t)$ has the following properties.

Lemma 3.6. Let $\delta_{1}=\left|v_{+}-v_{*}\right|+\left|u_{+}-u_{*}\right|+\left|\theta_{+}-\theta_{*}\right|$, then $\left(V_{3}, U_{3}, \Theta_{3}\right)(\xi, t)$ satisfies, if $q \geq p$,

i. $\quad U_{3 \xi}(x, t) \geq 0,\left|U_{3 \xi}\right| \leq C \varepsilon \delta_{1}$, for $t \geq 0, \xi>0$,

ii. For any $p(1 \leq p \leq+\infty)$, there exists a constant $C_{p, q}$ such that

$\left\|\left(V_{3 \xi}, U_{3 \xi}, \Theta_{3 \xi}\right)\right\|_{L^{p}(\xi \geq 0)} \leq C_{p, q} \min \left\{\delta_{1} \varepsilon^{1-\frac{1}{p}}, \delta_{1}^{\frac{1}{p}}(1+t)^{-1+\frac{1}{p}}\right\}$,

$\left\|\left(V_{3 \xi \xi}, U_{3 \xi \xi}, \Theta_{3 \xi \xi}\right)\right\|_{L^{p}(\xi \geq 0)} \leq C_{p, q} \min \left\{\delta_{1} \varepsilon^{2-\frac{1}{p}}, \delta_{1}^{\frac{1}{q}}(1+t)^{-1+\frac{1}{q}}\right\}, \quad t \geq 0$.

iii. $\left.\quad(V, U, \Theta)\right|_{\xi \leq-k_{-} t}=\left(v_{*}, u_{*}, \theta_{*}\right),\left.\left(V_{3 \xi}, U_{3 \xi}, \Theta_{3 \xi}, V_{3 \xi \xi}, U_{3 \xi \xi}, \Theta_{3 \xi \xi}\right)\right|_{\xi \leq-k_{-} t}=0$.

iv. $\limsup _{t \rightarrow+\infty, \xi \in\{\xi \geq 0\}}\left|(V, U, \Theta)(\xi, t)-\left(v^{r}, u^{r}, \theta^{r}\right)(\xi, t)\right|=0$. 
Let

and

$$
\left(\begin{array}{c}
V \\
U \\
\Theta
\end{array}\right)(\xi, t)=\left(\begin{array}{c}
V_{B}(\xi)+V_{3}(\xi, t)-v_{*} \\
U_{B}(\xi)+U_{3}(\xi, t)-u_{*} \\
\Theta_{B}(\xi)+\Theta_{3}(\xi, t)-\theta_{*}
\end{array}\right),
$$

$$
(\phi, \psi, \theta)(\xi, t)=(v-V, u-U, \theta-\Theta)(\xi, t) .
$$

Then the system (1.6) is rewritten as

$$
\left\{\begin{array}{l}
\phi_{t}-k_{-} \phi_{\xi}-\psi_{\xi}=0, \\
\psi_{t}-k_{-} \psi_{\xi}+[p(v, \theta)-p(V, \Theta)]_{\xi}=\mu\left(\frac{u_{\xi}}{v}\right)_{\xi}-\mu\left(\frac{U_{\xi}}{V}\right)_{\xi}+F_{\xi} \\
\zeta_{t}-k_{-} \zeta_{\xi}+\frac{\theta p_{\theta}}{e_{\theta}} \psi_{\xi}+\left\{\frac{\theta p_{\theta}}{e_{\theta}}(v, \theta)-\frac{\Theta p_{\theta}}{e_{\theta}}(V, \Theta)\right\} U_{\xi} \\
=\frac{\left\{\left(\frac{\kappa \theta_{\xi}}{v}\right)_{\xi}+\frac{\mu u_{\xi}^{2}}{v}\right\}}{e_{\theta}(v, \theta)}-\frac{\left\{\left(\frac{\kappa \Theta_{\xi}}{V}\right)_{\xi}+\frac{\mu U_{\xi}^{2}}{V}\right\}}{e_{\theta}(V, \Theta)}+G \\
\left.(\phi, \psi, \zeta)\right|_{\xi=0}=(0,0,0), \\
\left.(\phi, \psi, \zeta)\right|_{t=0}=(v-V, u-U, \theta-U)(\xi, 0)=:\left(\phi_{0}, \psi_{0}, \zeta_{0}\right)(\xi)
\end{array}\right.
$$

where

$$
\begin{aligned}
F= & -\left[p(V, \Theta)-p\left(V_{B}, \Theta_{B}\right)-p\left(V_{3}, \Theta_{3}\right)+p\left(v_{*}, \theta_{*}\right)\right]+\mu\left(\frac{U_{\xi}}{V}-\frac{U_{B \xi}}{V_{B}}\right) \\
= & :-F_{1}+F_{2}, \\
G= & -\left[\frac{\Theta p_{\theta}}{e_{\theta}}(V, \Theta) U_{\xi}-\frac{\Theta_{B} p_{\theta}}{e_{\theta}}\left(V_{B}, \Theta_{B}\right) U_{B \xi}-\frac{\Theta_{3} p_{\theta}}{e_{\theta}}\left(V_{3}, \Theta_{3}\right) U_{3 \xi}\right] \\
& +\left\{\frac{\left[\left(\frac{\kappa \Theta_{\xi}}{V}\right)_{\xi}+\frac{\mu U_{\xi}^{2}}{V}\right]}{e_{\theta}(V, \Theta)}-\frac{\left[\left(\frac{\kappa \Theta_{B \xi}}{V_{B}}\right)_{\xi}+\frac{\mu U_{B \xi}^{2}}{V_{B}}\right]}{e_{\theta}\left(V_{B}, \Theta_{B}\right)}\right\}=:-G_{1}+G_{2} .
\end{aligned}
$$

The equation (3.37) is almost the same as (3.3) except for the terms $F_{\xi}$ and $G$. Since

$$
\begin{aligned}
\left|F_{1 \xi}\right| \leq & C\left(\left|V_{3}-v_{*}\right|+\left|\Theta_{3}-\theta_{*}\right|\right)\left(\left|V_{B \xi}\right|+\left|\Theta_{B \xi}\right|\right) \\
& +C\left(\left|V_{B}-v_{*}\right|+\left|\Theta_{B}-\theta_{*}\right|\right)\left(\left|V_{3 \xi}\right|+\left|\Theta_{3 \xi}\right|\right),
\end{aligned}
$$

and $V_{3 \xi}=\Theta_{3 \xi}=V_{3}-v_{*}=\Theta_{3}-\theta_{*}=0$ for any $\xi<-k_{-} t$ duo to Lemma 3.6, we have

$$
\begin{aligned}
& \int_{0}^{\infty}\left|F_{1 \xi}\right| d \xi \leq C \delta_{1} \int_{-k_{-} t}^{\infty}\left|V_{B \xi}\right|+\left|\Theta_{B \xi}\right|+\left|V_{B}-v_{*}\right|+\left|\Theta_{B}-\theta_{*}\right| d \xi \\
\leq & C \delta \delta_{1} e^{-k_{-} t} .
\end{aligned}
$$

Thus

$$
\int_{0}^{t} \int_{0}^{+\infty}\left|F_{1 \xi} \psi\right| d \xi d t \leq C \delta \delta_{1} .
$$


On the other hand, since

$$
\begin{aligned}
\left|F_{2 \xi}\right| \leq & C\left(\left|V_{3}-v_{*}\right|\left|U_{B \xi \xi}\right|+\left|U_{3 \xi \xi}\right|+\left|V_{3 \xi} U_{3 \xi}\right|+\left|V_{3 \xi} U_{B \xi}\right|\right. \\
& \left.+\left|U_{3 \xi} V_{B \xi}\right|+\left|V_{3}-v_{*}\right|\left|V_{B \xi}\right|\right),
\end{aligned}
$$

we have

$$
\int_{0}^{\infty}\left|F_{2 \xi}\right| d \xi \leq C \delta \delta_{1} e^{-k_{-} t}+C \delta_{1}^{\frac{1}{8}}(1+t)^{-\frac{7}{8}}
$$

and then

$$
\int_{0}^{t} \int_{0}^{\infty}\left|F_{2 \xi} \psi\right| d \xi d t \leq C \delta \delta_{1}+\nu \int_{0}^{t}\left\|\psi_{\xi}\right\|^{2} d t+C_{\nu} \delta_{1}^{\frac{1}{6}}
$$

for any $\nu>0$. The terms $\int_{0}^{t} \int_{0}^{\infty}\left|G_{1} \psi\right| d \xi d \tau$ and $\int_{0}^{t} \int_{0}^{\infty}\left|G_{2} \psi\right| d \xi d \tau$ can be treated in the same way. Using the same argumens as in section 3.1, we obtain

$$
\begin{aligned}
& \|(\phi, \psi, \zeta)(t)\|^{2}+\int_{0}^{t}\left\|\partial_{\xi}(\psi, \zeta)(\tau)\right\|^{2} d \tau \\
\leq & C\left(\|(\phi, \psi, \zeta)(0)\|_{1}^{2}+\delta \int_{0}^{t}\left\|\phi_{\xi}\right\|^{2} d \tau+\delta+\delta_{1}^{\frac{1}{6}}\right) .
\end{aligned}
$$

The high order estimates are omitted here because they are similar to those in section 3.1. Therefore we have

THEOREM 3.7. Suppose that $\left(\phi_{0}, \psi_{0}, \zeta_{0}\right) \in H_{0}^{1}\left(R_{+}\right)$. Then there exists a positive constant $\delta_{0}$ such that if

$$
\left\|\left(\phi_{0}, \psi_{0}, \zeta_{0}\right)\right\|_{1}+\delta+\delta_{1} \leq \delta_{0}
$$

then the initial boundary value problem (3.37) has a unique global solution in time satisfying

$$
\left\{\begin{array}{l}
(\phi, \psi, \zeta) \in C^{0}\left(0,+\infty ; H_{0}^{1}\right), \\
\phi_{\xi} \in L^{2}\left(0,+\infty ; L^{2}\right), \\
(\psi, \zeta)_{\xi} \in L^{2}\left(0,+\infty ; H^{1}\right),
\end{array}\right.
$$

and

$$
\limsup _{t \rightarrow+\infty, \xi>0}|(\phi, \psi, \zeta)(\xi, t)| \rightarrow 0
$$

REMARK 3.1. The case $\left(v_{-}, u_{-}, \theta_{-}\right) \in R_{1} R_{3}\left(v_{+}, u_{+}, \theta_{+}\right) \cap \Omega_{\text {super }}$ can be treated in a similar way and then the assertion (III) holds.

Acknowledgement. The authors wish to thank Prof. Akitaka Matsumura for his many helpful discussions and kind hospitality. The work of F. Huang was supported in part by the JSPS Research Fellowship for foreign researchers and Grandin-aid No.P-00269 for JSPS from the ministry of Education, Science, Sports, and Culture of Japan. 


\section{REFERENCES}

[1] F. Huang, A. Matsumura and X. Shi, A gas-solid free boundary problem for compressible viscous gas, SIAM J. Math. Anal., 34(6), 1331-1355, 2003.

[2] F. Huang, A. Matsumura and X. Shi, On the stability of contact discontinuity for compressible Navier-Stokes equations with free boundary, Osaka J. Math., 41(1), 193-210, 2004.

[3] S. Kawashima and A. Matsumura, Asymptotic stability of traveling wave solutions of systems for one-dimensional gas motion, Commun. Math. Phys., 101, 97-127, 1985.

[4] S. Kawashima, A. Matsumura and K. Nishihara, Asymptotic behavior of solutions for the equations of a viscous heat-conductive gas, Proc. Japan Acad., Ser.A, 62, 249-252, 1986.

[5] A. Kelley, The stable, center-stable, center, center-unstable, unstable manifolds, J. Diff. Equs., 3, 546-570, 1967.

[6] Y. Nikkuni and S. Kawashima, Stability of stationary solutions to the half-space problem for the discrete Boltzmann equation with multiple collisions, Kyushu J. Math., 54(2), 233-255, 2000 .

[7] T. Liu, A. Matsumura and K. Nishihara, Behaviors of solutions for the Burgers equation with boundary corresponding to rarefaction waves, SIAM J. Math. Anal., 29, 293-308, 1998.

[8] A. Matsumura, Inflow and outflow problems in the half space for a one-dimensional isentropic model system of compressible viscous gas, IMS Conference on Differential Equations from Mechanics (Hong Kong, 1999). Methods Appl. Anal., 8(4), 645-666, 2001.

[9] A. Matsumura and K. Nishihara, On the stability of traveling wave solutions of a onedimensional model system for compressible viscous gas, Japan J. Appl. Math., 2, 17-25, 1985.

[10] A. Matsumura and K. Nishihara, Asymptotics toward the rarefaction wave of the solutions of a one-dimensional model system for compressible viscous gas, Japan J. Appl. Math., 3, $1-13,1986$.

[11] A. Matsumura and K. Nishihara, Global stability of the rarefaction wave of a one-dimensional model system for compressible viscous gas, Commun. Math. Phys., 144, 325-335, 1992.

[12] A. Matsumura and K. Nishihara, Global asymptotics toward the rarefaction wave for solutions of viscous p-system with boundary effect, Q. Appl. Math., LVIII, 69-83, 2000.

[13] A. Matsumura and K. Nishihara, Large time behaviors of solutions to an inflow problem in the half space for a one-dimensional system of compressible viscous gas, Commun. Math. Phys., 222(3), 449-474, 2001.

[14] S. Nishibata, S. Kawashima and P. Zhu, Asymptiotic stbility of stationary solution to the compressible Navier-Stokes equations in the half space, Commun. Math. Phys., 240(3), 483-500, 2001.

[15] X. Shi, On the stability of rarefaction wave solutions for viscous p-system with boundary effect, Acta. Math. Appl. Sin. Engl. Ser., 19(2), 341-352, 2003.

[16] S. Kawashima and P.Zhu, Existence and asymptotic stability of stationary solution to the full compressible Navier-Stokes equations in the half space, Mathematical analysis in fluid and gas dynamics, RIMS kokyuroku 1247, 187-207, 2002. 